\title{
Implementation in partial equilibrium
}

\author{
Takashi Hayashi *, Michele Lombardi \\ University of Glasgow, Adam Smith Business School, West Quadrangle, Gilbert Scott Building, Glasgow, G12 8QQ, \\ United Kingdom
}

Received 4 May 2016; final version received 11 January 2017; accepted 20 January 2017

Available online 27 January 2017

\begin{abstract}
Consider a society with a finite number of sectors (social issues or commodities). In a partial equilibrium (PE) mechanism a sector authority (SA) aims to elicit agents' preference rankings for outcomes at hand, presuming separability of preferences, while such presumption is false in general and such isolated rankings might be artifacts. This paper studies what can be Nash implemented if we take such misspecification of $\mathrm{PE}$ analysis as a given institutional constraint. The objective is to uncover the kinds of complementarity across sectors that this institutional constraint is able to accommodate. Thus, in our implementation model there are several SAs, agents are constrained to submit their rankings to each SA separately and, moreover, SAs cannot communicate with each other. When a social choice rule (SCR) can be Nash implemented by a product set of PE mechanisms, we say that it can be Nash implemented in PE. We identify necessary conditions for SCRs to be Nash implemented in PE and show that they are also sufficient under a domain condition which identifies the kinds of admissible complementarities. Thus, the Nash implementation in PE of SCRs is examined in auction and matching environments.

(c) 2017 The Author(s). Published by Elsevier Inc. This is an open access article under the CC BY-NC-ND license (http://creativecommons.org/licenses/by-nc-nd/4.0/).
\end{abstract}

JEL classification: D47; D71; D82

\footnotetext{
Wh are grateful to Salvador Barberà, Clemens Puppe, William Thomson and audiences at the Workshop on Social Choice and Mechanism Design (Manchester, 2015), the SIRE Behaviour, Incentives and Contracts Workshop (Edinburgh, 2015), the Workshop on Game Theory and Social Choice (Budapest, 2015), the European Winter Meeting of the Econometric Society (Milan, 2015), the European Meeting on Game Theory (SING12; Odense, 2016), the Meeting of the Society for Social Choice and Welfare (Lund, 2016), the World Congress of the Game Theory Society (Maastricht, 2016), Hitotsubashi University, Waseda University, Yokohama National University and the University of Cergy-Pontoise, for useful comments and suggestions. We are also grateful to an associate editor and two referees of this Journal for their useful comments and suggestions. The usual caveat applies.

* Corresponding author.

E-mail addresses: takashi.hayashi@glasgow.ac.uk (T. Hayashi), michele.lombardi@glasgow.ac.u (M. Lombardi).
} 
Keywords: Implementation; Partial equilibrium; Complementarity; Income effect

\section{Introduction}

The methodology used in the literature of mechanism design in order to understand how to solve a single allocation decision problem whose solution depends on private information held by various agents is that of PE analysis. This methodology isolates outcomes to be allocated as well as people's preferences for those outcomes from the rest of the world, under a ceteris paribus (all else equal) assumption. Because of such isolation, PE mechanism design has provided exact mechanisms and algorithms on how to elicit the private information from agents so as to achieve desirable allocation decisions, and has proved capable of handling a wide variety of issues, not only economic but also political and legal. The prominently successful cases are auction and matching.

Of course, this isolation is legitimate if agents have separable preferences over a product set of outcomes $X=X^{1} \times \cdots \times X^{\ell}$. This is because when an agent has a separable preference, a well-defined marginal preference exists on each component set $X^{s}$ of the product set, which is independent of the values of other components.

The ceteris paribus assumption, however, cannot be true in general, since people's preferences are generally non-separable. This means that a marginal preference over a component set depends on the values chosen for the other components. For example, which school one would like to be admitted to may depend on where she lives and, moreover, which catchment area she would like to live may depend on which school she could be admitted to. When the school authority requires participants to report their school rankings, it believes that its participants have separable preferences, while such rankings may be artifacts.

Not least, when we change something in the school admission program, it will have a general equilibrium effect, such as changes in the housing market and how people choose where to live, etc. Likewise, when we change something in an auction rule, it will have a general equilibrium effect on how people consume goods related to the auctioned item and, moreover, will affect bidders' willingness to pay for the item auctioned off, and so on.

Perhaps, a centralized allocation mechanism may be better equipped to deal with issues arising from non-separability of agents' preferences. However, this mechanism is not available or feasible in real life. Given that the goal of implementation theory is to study the relationship between outcomes in a society and the mechanisms under which those outcomes arise, it is important to throw light on how such isolations dictated by the practice of PE mechanisms affect outcomes in society. In this paper, we ask the following questions: What do we lose by ignoring such general equilibrium effects? More specifically, if we take the practice dictated by PE mechanisms as a given institutional constraint, can one describe the requirements on SCRs that are equivalent to Nash implementability by a product set of PE mechanisms? What kind of complementarity, if any, is this practice able to accommodate?

This paper answers the above questions by assuming that there are $\ell \geq 2$ social issues, or sectors, and $n \geq 3$ agents in society. It assumes that every agent in society is involved in all social issues.

Moreover, it supposes that there is a Central Authority (CA) who wishes to Nash implement a SCR, which depends on private information held by various agents. Since the CA cannot design any centralized mechanism and, thus, cannot elicit any private information from agents, he delegates the decision-making authority to independent SAs, such as the school authority, the 
housing authority, and so on. ${ }^{1}$ Thus, the CA cannot control the behaviour of agents. Instead, their interaction is controlled by independent SAs.

Given these delegation arrangements, a SA dealing with the social issue $s$ designs an allocation mechanism or PE mechanism, $\Gamma^{s}$, for the issue at hand. This mechanism asks agents to report only the information pertaining to the issue $s$ as well as assigns outcomes of $X^{s}$ on the basis of the information elicited from agents. Given a product set of PE mechanisms, one for each issue, denoted by $\Gamma=\Gamma^{1} \times \cdots \times \Gamma^{\ell}$, each agent communicates with each SA separately. Since each SA specifies the PE mechanism in advance, the agents themselves know exactly not only which game induced by $\Gamma^{s}$ is being played for the issue $s$, but also which overall game induced by $\Gamma$ is being played.

This paper uses Nash equilibrium as the equilibrium concept for solving the game that $\Gamma^{s}$ leads to in every environment and for solving the game that $\Gamma$ leads to. This is because difficulties arise when agents' preference are non-separable. For instance, a classic PE mechanism is the so-called Top-Trading-Cycle (TTC) algorithm (Shapley and Scarf, 1974). Many methods for finding desirable allocations in matching environments are variants of this algorithm. The reason for its success is that the TTC algorithm is strategy-proof; that is, true-telling about her own marginal preferences for houses (or some other indivisible items such as tasks or jobs) is a dominant strategy for each agent. However, when preferences are not separable, a dominant strategy no longer exists. Indeed, there is not even a "true" marginal preference for houses. With non-separable preferences it thus becomes necessary to consider a weaker notion of equilibrium.

In the standard literature of Nash implementation, a SCR is Nash implementable if the authority can design a mechanism whose set of Nash equilibrium outcomes coincides with the outcomes prescribed by the welfare criterion incorporated into the SCR. In our set up, the CA has the same objective as in the standard set up, only now he has to achieve it via a product set of PE mechanisms $\Gamma$. Moreover, SAs have the same objective as in the standard set up, only now their Nash implementation problems pertain only to their respective issues. Finally, the objectives of SAs are linked to the CA's objective.

As in Maskin's (1999) original result, we show that a SCR that can be Nash implemented in PE satisfies a remarkably strong invariance condition, now widely referred to as Maskin monotonicity. Furthermore, we also show that it satisfies a decomposability condition, a sector-wise Maskin monotonicity condition and a decomposable Maskin monotonicity condition.

Decomposability requires that the SCR can be decomposed into one-dimensional SCRs, one for each sector, and that the range of the SCR is the product of the ranges of the one-dimensional SCRs if the domain of the SCR consists only of separable preferences. Sector-wise Maskin monotonicity requires that each one-dimensional SCR needs to satisfy the standard invariance condition due to Maskin (1999). Decomposable Maskin monotonicity states that an outcome $\left(x^{1}, \cdots, x^{s-1}, x^{s}, x^{s+1} \cdots, x^{\ell}\right) \in X$ should continue to be selected by a given SCR at one profile of separable preferences $R^{\prime}$ if $\left(x^{1}, \cdots, x^{s-1}, x^{s}, x^{s+1} \cdots, x^{\ell}\right)$ has been one of the outcomes selected by the SCR at one preference profile $R$ and the state has moved from $R$ to $R^{\prime}$ in a such way that for no agent and for no sector $s$ it is true that $\left(x^{1}, \cdots, x^{s-1}, x^{s}, x^{s+1} \cdots, x^{\ell}\right)$ has fallen with respect to any alternative of the type $\left(x^{1}, \cdots, x^{s-1}, y^{s}, x^{s+1} \cdots, x^{\ell}\right)$ in her personal ranking.

\footnotetext{
1 In line with implementation literature, we use the term SA as an idiom for a social planner who selects the mechanism to implement a SCR and who only cares about the welfare of society.
} 
Given these necessary conditions, and under a domain restriction, that we have named $P 1$, we characterize Nash implementability in PE with recourse to two conditions reminiscent of the so-called no veto-power condition. It follows from this result that for Nash implementation problems in PE in which there is a private good, Nash implementability in PE of a SCR is nearly equivalent to sector-wise Maskin monotonicity, decomposability, decomposable Maskin monotonicity and Maskin monotonicity.

Our exercise does not deliver anything new when agents have separable preferences, because the implementation problem can simply be decomposed into several implementation problems, one for each sector, and each SA solves its problem separately. The interesting case is thus when preferences are not separable. What can we do in this case given the institutional constraint represented by the practice of PE mechanisms? Under the domain restriction P1, SAs do not need to care about non-separabilities, and the CA cannot do anything about it. School board simply asks households to submit rankings over schools, whether there is indeed such thing or not. Housing agency simply asks households to submit rankings only over houses, whether there is indeed such thing or not. Auction agency simply asks bidders to submit their willingness to pay for an item, whether there is indeed such thing or not, and so on. ${ }^{2}$

To illustrate our answer further, in a perhaps cynical manner, consider that there are two sectors, say $L$ and $R$, where sector $L$ allocates left shoes and sector $R$ allocates right shoes. When an agent reports to the SA for $L$ that she wants some left shoe, we can naturally understand that this is so because she intends and expects to get the right shoe counterpart from SA for $R$. However, the SA for $L$ here understands only that she likes that left shoe as an individual item, and does not question why she wants it. This is stupid, but this is exactly the nature of PE mechanism design.

The domain restriction $\mathrm{P} 1$ roughly states that one can never gain by getting worse outcome in each sector. This restricts the kinds of complementarities that are admissible in PE design. One might suspect that this domain condition is simply a tailor-made one such that the product of sector-wise mechanisms works. However, we show that the condition is indispensable for any PE mechanism to work, not just for the particular mechanism we have constructed (see Example 1). Therefore, in order to accommodate complementarities that lay outside our domain condition there is a need to move away from the practice of PE analysis and to start analyzing implementation problems where an incomplete, but yet not negligible, communication is allowed among SAs and where the CA has to make some modelling choice about how SAs communicate.

Section 4 assesses the implications of our characterization result in matching and auction settings. It shows that some non-dictatorial SCRs defined on preference domains that allow nonseparability of preferences are Nash implementable in PE. For instance, in a multi-item auction setting with private values in which buyers have non-separable preferences for items being sold due to income effects, one can attain the goal of efficiency as a Nash equilibrium outcome in PE by means of the sector-wise Vickrey (second-price; 1961) auction solution. Roughly speaking,

\footnotetext{
2 We should note that in our discussion we say each individual reports only marginal rankings to each SA just for illustration purpose. Actually, for each of the mechanism we construct, individuals' strategies also include an allocation component and tie-breaking device component. Given that our mechanisms are admittedly abstract and there is no reason to restrict attention to them, one may wonder whether one can obtain more permissive results if each individual is allowed to report information about the entire economy. Unfortunately, the result cannot change because of the institutional constraint of the PE analysis. Indeed, what an individual may report to one SA about the entire economy can be different from what she reports to another SA. Moreover, the SAs do not communicate each other for cross-checking the information collected and there is no additional informational value in doing so.
} 
the sector-wise Vickrey auction solution is a solution that assigns each item to the highest bidder and prescribes that this winner pays the amount of the second-highest bid.

The remainder of the paper is organized as follows. Section 2 sets out the theoretical framework and outlines the basic model, while necessary and sufficient conditions are presented in section 3. Section 4 assesses the implications of our characterization result. Section 5 concludes by suggesting directions for future research. Appendix includes proofs not in the main body.

\section{Preliminaries}

We consider a finite set of agents indexed by $i \in I=\{1, \cdots, n\}$ and a finite set of elementary sectors indexed by $s \in S=\{1, \cdots, \ell\}$. The set of outcomes of sector $s$ available to agents is represented by $X^{s}$, with $x^{s}$ as a typical element. $X^{s}$ is called sector-s outcome space. We assume that the set of outcomes available to agents is the product space

$$
X=\prod_{s \in S} X^{s}
$$

To economize on notation, for any sector $s$, write $s_{C}$ for the complement of $s$ in $S$. Thus, $\left(x^{s}, x^{s_{C}}\right)$ is an outcome of $X$, where it is understood that $x^{s_{C}}$ is an element of the product space $X^{s_{C}}=\prod_{\bar{s} \in s_{C}} X^{\bar{s}}$.

In the usual fashion, agent $i$ 's preferences over $X$ are given by a complete and transitive binary relation, subsequently an ordering, $R_{i}$ on $X$. The corresponding strict and indifference relations are denoted by $P\left(R_{i}\right)$ and $I\left(R_{i}\right)$, respectively. The statement $x R_{i} y$ means that agent $i$ judges $x$ to be at least as good as $y$. The statement $x P\left(R_{i}\right) y$ means that agent $i$ judges $x$ better than $y$. Finally, the statement $x I\left(R_{i}\right) y$ means that agent $i$ judges $x$ and $y$ as equally good.

The condition of separability of preferences that must hold if the isolation of sector- $s$ decision problem from others is legitimate can be formulated as follows. For each $x^{s_{C}}$, we define the sector-s marginal ordering, $R_{i}^{s}\left(x^{s_{C}}\right)$, on $X^{s}$ by

$$
\text { for all } y^{s}, z^{s} \in X^{s}: y^{s} R_{i}^{s}\left(x^{s_{C}}\right) z^{s} \Longleftrightarrow\left(y^{s}, x^{s_{C}}\right) R_{i}\left(z^{s}, x^{s_{C}}\right) \text {. }
$$

We say that the ordering $R_{i}$ is separable if for all $s \in S$,

$$
R_{i}^{S}\left(x^{s_{C}}\right)=R_{i}^{s}\left(y^{s_{C}}\right) \text { for all } x^{s_{C}}, y^{s_{C}} \in X^{s_{C}} .
$$

In other words, $R_{i}$ is separable if the agent $i$ 's preferences over outcomes of $X^{s}$ are independent of outcomes chosen from $X^{s_{C}}$. Again, to save writing, for any separable ordering $R_{i}$, write $R_{i}^{s}$ for the sector- $s$ marginal ordering induced by $R_{i}$.

We assume that the CA does not know agent $i$ 's true preferences. Thus, write $\mathcal{R}(X)$ for the set of orderings on $X, \mathcal{R}^{s e p}(X)$ for the set of separable orderings on $X$, and $\mathcal{R}_{i}$ for the domain of (allowable) orderings on $X$ for agent $i$. We assume that the CA knows the domain of preferences for the set $I$, which is the product set of agents' domains, that is,

$$
\mathcal{R}_{I}=\prod_{i \in I} \mathcal{R}_{i},
$$

with $R$ as a typical profile. Also, we assume that there is complete information among the agents in $I$. 


\subsection{PE mechanisms}

The goal of the CA is to implement a $\operatorname{SCR} \varphi: \mathcal{R}_{I} \rightarrow X$ where $\varphi(R)$ is nonempty for any $R \in \mathcal{R}_{I}$. We shall refer to $x \in \varphi(R)$ as a $\varphi$-optimal outcome at $R$. The common interpretation is that a SCR represents the social objectives that the society or its representatives want to achieve.

The CA delegates the achievement of the goal(s) to SAs, each of which believes that agents have separable preferences and, subsequently, designs a PE mechanism. Because we endorse the methodology of PE analysis, each SA is assumed to be able to conceive only marginal preferences which are consistent with allowable separable preferences. Formally, for each $s \in S$, we denote sector- $s$ domain for agent $i$ by $\mathcal{D}_{i}^{s}$, which is defined by

$$
\left\{R_{i}^{s} \mid R_{i}^{s} \text { is induced by } R_{i} \in \mathcal{R}_{i} \bigcap \mathcal{R}^{s e p}(X)\right\}=\mathcal{D}_{i}^{s} .
$$

To see why this is needed, consider a multi-item auction setting with private values in which buyers have preferences for items being sold and where each item is auctioned separately. In this setting, any allowable preference cares for the sum of income transfers across sectors, and cash transfers are not to be evaluated differently: money is money. Because of this, any allowable separable preference has to be represented in the form of a sum of quasi-linear functions under the methodology of PE analysis. Then, given that marginal orderings induced by non-separable preferences are not necessarily quasi-linear, the SA will notice that there is something wrong with the methodology when he perceives that participants may have non-quasi-linear preferences for outcomes of his sector. In order that PE mechanism design works "successfully," such type of situations have to be avoided.

Write $\mathcal{D}_{I}^{s}$ for the product set of $\mathcal{D}_{i}^{s}$, s, with $R^{s}$ as a typical profile. The goal of sector- $s$ SA is to implement a one-dimensional SCR $\varphi^{s}: \mathcal{D}_{I}^{s} \rightarrow X^{s}$ where $\varphi^{s}\left(R^{s}\right)$ is nonempty for any $R^{s} \in \mathcal{D}_{I}^{s}$. Again, we shall refer to $x^{s} \in \varphi^{s}\left(R^{s}\right)$ as a $\varphi^{s}$-optimal sector-s outcome at $R^{s}$.

The delegated sector- $s$ SA knows the domain of sector-s preferences $\mathcal{D}_{I}^{s}$ and the delegated objective $\varphi^{s}$. However, we assume that this authority is unable to associate any element of $\mathcal{D}_{i}^{s}$ with a specific element of agent $i$ 's domain $\mathcal{R}_{i}$. To make the analysis consistent with the methodology of PE analysis we further assume throughout the paper that (i) the only concern of a SA is to promote the goal(s) of the CA and (ii) there is no communication between SAs about the information elicited from the agents.

Each SA delegates the choice to agents according to a PE mechanism, which aims to elicit the private information related to sector $s$ from agents. Thus, in pursuing his target(s), sector- $s$ SA designs a PE mechanism $\Gamma^{s}=\left(\left(M_{i}^{s}\right)_{i \in I}, h^{s}\right)$, where $M_{i}^{s}$ is the strategy space of agent $i$ in sector $s$ and $h^{s}: M^{s} \rightarrow X^{s}$, the outcome function, assigns to every strategy profile

$$
m^{s} \in M^{s}=\prod_{i \in I} M_{i}^{s}
$$

a unique outcome in $X^{s}$.

A PE mechanism $\Gamma^{s}$ together with the profile $R^{s} \in \mathcal{D}_{I}^{s}$ defines a strategic game $\left(\Gamma^{s}, R^{s}\right)$ in sector $s$, in which each agent chooses her strategy and all agents' strategy choices are made simultaneously (that is, when choosing a strategy choice each agent is not informed of the strategy choice chosen by any other agent). A strategy profile $m^{s} \in M^{s}$ is a Nash equilibrium (in pure strategies) of $\left(\Gamma^{s}, R^{s}\right)$ if for all $i \in I$, it holds that

$$
\text { for all } \bar{m}_{i}^{s} \in M_{i}^{s}: h^{s}\left(m^{s}\right) R_{i}^{s} h^{s}\left(\bar{m}_{i}^{s}, m_{-i}^{s}\right) \text {. }
$$


Write $N E\left(\Gamma^{s}, R^{s}\right)$ for the set of Nash equilibrium profiles of $\left(\Gamma^{s}, R^{s}\right)$, and write $h^{s}\left(N E\left(\Gamma^{s}, R^{s}\right)\right)$ for the set of Nash equilibrium outcomes of $\left(\Gamma^{s}, R^{s}\right)$.

In delegating the achievement of the goal(s) to SAs, the CA 'loses control' of the mechanism design exercise. In other words, he does not design any mechanism. Moreover, from his point of view, each agent is free to choose strategically from her strategy space $M_{i}=\prod_{s \in S} M_{i}^{s}$ so as to influence the outcomes of PE mechanisms in her favour. Naturally, which outcomes can be obtained by agent $i$ depends on profiles of outcomes that this agent can achieve in each sector $s$, while keeping her opponents' actions fixed at some strategy profile $m_{-i} \in \prod_{j \in I \backslash\{i\}}\left(\prod_{s \in S} M_{j}^{s}\right)$. Therefore, from the point of view of the CA, the mechanism governing communication with agents is a product set of PE mechanisms $\Gamma=\left(\left(M_{i}\right)_{i \in N}, h\right)$, where $M_{i}$ is the strategy space of agent $i$ and $h: M \rightarrow X$, the outcome function, assigns to every strategy profile

$$
m \in M=\prod_{i \in I} M_{i}
$$

a unique outcome in $X$ such that

$$
h(m)=\left(h^{s}\left(m^{s}\right)\right)_{s \in S} .
$$

A product set of PE mechanisms $\Gamma \equiv\left(\Gamma^{s}\right)_{s \in S}$ and a profile $R \in \mathcal{R}_{I}$ induce a strategic game $(\Gamma, R)$. A strategy profile $m \in M$ is a Nash equilibrium (in pure strategies) of $(\Gamma, R)$ if for all $i \in I$, it holds that

$$
\text { for all } \bar{m}_{i} \in M_{i}: h(m) R_{i} h\left(\bar{m}_{i}, m_{-i}\right) \text {, }
$$

where, as usual, $m_{-i}$ is the strategy profile of all agents except $i$ such that $\left(m_{i}, m_{-i}\right)=m$. Write $N E(\Gamma, R)$ for the set of Nash equilibrium profiles of $(\Gamma, R)$, and write $h(N E(\Gamma, R))$ for the set of Nash equilibrium outcomes of $(\Gamma, R)$.

\subsection{Nash implementation in $P E$}

Suppose that the CA wants to Nash implement the SCR $\varphi$. In the standard literature, the CA provides agents with a mechanism which has the following feature. For every admissible profile of orderings, the set of Nash equilibrium outcomes of the mechanism for that profile is identical to the set of outcomes dictated by the SCR for it. In our set up, the CA has the same objective as in the standard set up, only now he has to achieve it via a product set of PE mechanisms; that is, via a mechanism where the decision-making authority is delegated to SAs (see part (i) of Definition 1 below). Moreover, SAs have the same objective as in the standard set up, only now their Nash implementation problems pertain only to their respective sectors (see part (ii) of Definition 1 below). Finally, if the delegation from the CA to SAs is to be effective SAs' objectives must be linked to the CA's objective. Thus, and in contrast to the standard definition of Nash implementation, our definition of Nash implementation in PE embodies the existence of a structure which links the goals of SAs to the CA's goal (see part (iii) of Definition 1 below). A sector-s SA cannot do its part unless it receives information telling it what outcomes are to be achieved as Nash equilibrium outcomes for the domain $\mathcal{D}_{I}^{s}$. Moreover, if $x$ is a Nash equilibrium outcome at 
state $R$ but for no element of the domain $\mathcal{D}_{I}^{s}$ the outcome $x^{s}$ can be obtained as a sector- $s$ Nash equilibrium outcome, the SA would be able to detect problems with the domain $\mathcal{D}_{I}^{s}{ }^{3}$

Definition 1. The SCR $\varphi: \mathcal{R}_{I} \rightarrow X$ is Nash implementable in PE if there exist a product set of PE mechanisms $\Gamma$ and a sequence $\left(\varphi^{s}\right)_{s \in S}$ of one-dimensional SCRs, where $\varphi^{s}: \mathcal{D}_{I}^{s} \rightarrow X^{s}$ for all $s \in S$, such that:

(i) for all $R \in \mathcal{R}_{I}: \varphi(R)=h(N E(\Gamma, R))$,

(ii) for all $s \in S: \varphi^{s}\left(R^{s}\right)=h^{s}\left(N E\left(\Gamma^{s}, R^{s}\right)\right)$ for all $R^{s} \in \mathcal{D}_{I}^{s}$,

(iii) for all $R \in \mathcal{R}_{I}: m \in N E(\Gamma, R)$ for some $m \in M \Longrightarrow m^{s} \in N E\left(\Gamma^{s}, \bar{R}^{s}\right)$ for some $\bar{R}^{s} \in \mathcal{D}_{I}^{s}$, for all $s \in S$.

Let $\Gamma$ be a product set of PE mechanisms. If a profile $R$ consists of separable orderings, then the Nash equilibrium outcomes of the game $\left(\Gamma^{s}, R^{s}\right)$ do not depend on outcomes that agents can obtain from games played in other sectors. Indeed, for cases like this, the Cartesian product of Nash equilibrium outcomes of the games $\left(\left(\Gamma^{s}, R^{s}\right)\right)_{s \in S}$ constitutes the set of Nash equilibrium outcomes of the game $(\Gamma, R)$; that is, $N E(\Gamma, R)=\prod_{s \in S} N E\left(\Gamma^{s}, R^{s}\right) .{ }^{4}$ Indeed, if the domain $\mathcal{R}_{I}$ of the $\operatorname{SCR} \varphi$ is represented by the domain of profiles of separable preferences, Nash implementation in PE consists only of part (ii) of the above definition.

\section{Necessary and sufficient conditions}

\subsection{Necessary conditions}

The relevance of implementation theory comes from the fact that it provides a theoretical construct within which to study the way in which a society shall trade off agent preferences to achieve its goals. Unless the SCR is dictatorial, this involves a compromise. The first condition identifies a property of how a SCR must handle the compromise across sectors where agents' preferences are separable. ${ }^{5}$

Definition 2. The SCR $\varphi: \mathcal{R}_{I} \rightarrow X$ is decomposable provided that for each $s \in S$, there exists a (nonempty) correspondence $\varphi^{s}: \mathcal{D}_{I}^{s} \rightarrow X^{s}$. Moreover, $\varphi(R)=\prod_{s \in S} \varphi^{s}\left(R^{s}\right)$ for each profile $R \in \mathcal{R}_{I} \bigcap \mathcal{R}_{I}^{\text {sep }}(X)$.

This says that if a SCR is decomposable, then the $s$ th dimension of the SCR depends only on the profiles of marginal orderings of the $s$ th sector. Differently put, the SCR can be decomposed into the product of one-dimensional SCRs. Furthermore, it implies that the social objectives that a society or its representatives wants to achieve can be decomposed in 'small' social objectives, one for each sector. Therefore, to analyze the way in which the society should trade off agent preferences for the $s$ th sector to achieve its goal, we can ignore consumption trade-offs across sectors and focus only on the profiles of marginal orderings of $s$ th sector.

\footnotetext{
3 We are grateful to a referee and an associate editor for having pointed out the need of making explicit the link between objectives.

4 The proof is available upon request from the authors.

5 As usual, $\mathcal{R}_{I}^{\text {sep }}(X)=\mathcal{R}^{\text {sep }}(X) \times \ldots \times \mathcal{R}^{\text {sep }}(X)$ ( $n$ times).
} 
Theorem 1. The SCR $\varphi: \mathcal{R}_{I} \rightarrow X$ is decomposable if $\varphi$ is Nash implementable in PE.

Proof. Let the premises hold. Then, by Definition 1, there exists a product set of PE mechanisms $\Gamma$ and a sequence $\left(\varphi^{s}\right)_{s \in S}$ of one-dimensional SCRs, where $\varphi^{s}: \mathcal{D}_{I}^{s} \rightarrow X^{s}$ for all $s \in S$, such that parts (i)-(ii) are satisfied. Furthermore, take any profile $R \in \mathcal{R}_{I} \cap \mathcal{R}_{I}^{\text {sep }}(X)$. Note that

$$
\prod_{s \in S} N E\left(\Gamma^{s}, R^{s}\right)=N E(\Gamma, R) .
$$

Suppose that $x \in \varphi(R)=h(N E(\Gamma, R))$. By definition of the domain $\mathcal{D}_{i}^{s}$, it follows that the sector- $s$ marginal ordering $R_{i}^{s}$ induced by the separable ordering $R_{i}$ is an element of $\mathcal{D}_{i}^{s}$. Since $x \in h(N E(\Gamma, R))=\prod_{s \in S} h^{s}\left(N E\left(\Gamma^{s}, R^{s}\right)\right)$, part (ii) implies that $x^{s} \in \varphi^{s}\left(R^{s}\right)$ for all $s \in S$, as sought.

Conversely, suppose that $x^{s} \in \varphi^{s}\left(R^{s}\right)$ for all $s \in S$. Part (ii) implies that $x^{s} \in h^{s}\left(N E\left(\Gamma^{s}, R^{s}\right)\right)$ for all $s \in S$. Since $\prod_{s \in S} h^{s}\left(N E\left(\Gamma^{s}, R^{s}\right)\right)=h(N E(\Gamma, R))$, part (i) implies that $x \in \varphi(R)$. We conclude that $\varphi$ is decomposable.

In the literature of strategy-proof social choice functions, it has been shown that decomposability is implied by strategy-proofness where agents have separable preferences (as per Barberà et al., 1991; Le Breton and Sen, 1999). A natural question, then, is whether decomposability is implied by Nash implementation. ${ }^{6}$ The answer is no. ${ }^{7}$

A condition that is central to the Nash implementation of SCRs is Maskin monotonicity. This condition says that if an outcome $x$ is $\varphi$-optimal at the profile $R$ and this $x$ does not strictly fall in preference for anyone when the profile is changed to $R^{\prime}$, then $x$ must remain a $\varphi$-optimal outcome at $R^{\prime}$. Let us formalize that condition as follows. For any ordering $R_{i}$ and outcome $x$, the weak lower contour set of $R_{i}$ at $x$ is defined by $L\left(x, R_{i}\right)=\left\{x^{\prime} \in X \mid x R_{i} x^{\prime}\right\}$. Therefore:

Definition 3. The SCR $\varphi: \mathcal{R}_{I} \rightarrow X$ is Maskin monotonic provided that for all $x \in X$ and all $R, R^{\prime} \in \mathcal{R}_{I}$, if $x \in \varphi(R)$ and $L\left(x, R_{i}\right) \subseteq L\left(x, R_{i}^{\prime}\right)$ for all $i \in I$, then $x \in \varphi\left(R^{\prime}\right)$.

Theorem 2. The SCR $\varphi: \mathcal{R}_{I} \rightarrow X$ is monotonic if $\varphi$ is Nash implementable in PE.

Proof. The proof follows directly from Maskin (1999).

Furthermore, as one would expect, we require Maskin monotonicity for each sector; that is:

Definition 4. The decomposable $\operatorname{SCR} \varphi: \mathcal{R}_{I} \rightarrow X$ is sector-wise Maskin monotonic provided that for all $s \in S$, all $x^{s} \in X^{s}$ and all $R^{s}, \bar{R}^{s} \in \mathcal{D}_{I}^{s}$ if $x^{s} \in \varphi^{s}\left(R^{s}\right)$ and $L\left(x^{s}, R_{i}^{s}\right) \subseteq L\left(x^{s}, \bar{R}_{i}^{s}\right)$ for all $i \in I$, then $x^{s} \in \varphi^{s}\left(\bar{R}^{s}\right)$.

Theorem 3. The decomposable SCR $\varphi: \mathcal{R}_{I} \rightarrow X$ is sector-wise Maskin monotonic if $\varphi$ is Nash implementable in PE.

Proof. The proof can be found in Maskin (1999).

$\overline{{ }^{6} \mathrm{~A} \mathrm{SCR}} \varphi: \mathcal{R}_{I} \rightarrow X$ is Nash implementable if there exists a mechanism $\gamma \equiv(M, h)$ such that for all $R \in \mathcal{R}_{I}$, $\varphi(R)=h(N E(\gamma, R))$.

7 An example is available upon request from the authors. 
An equivalent statement of Maskin monotonicity stated above follows the reasoning that if $x$ is $\varphi$-optimal at $R$ but not $\varphi$-optimal at $R^{\prime}$, then the outcome $x$ must have fallen strictly in someone's ordering at the profile $R^{\prime}$ in order to break the Nash equilibrium via some deviation. Therefore, there must exist some preference reversal if an equilibrium strategy profile at $R$ is to be broken at $R^{\prime}$. When the new profile $R^{\prime}$ satisfies the requirement of separability and the $\operatorname{SCR} \varphi$ is Nash implementable in PE, then the $s$ th dimension of the SCR depends only on the profiles of marginal orderings of the $s$ th sector. Therefore, a variant of Maskin monotonicity follows the reasoning that if $x$ is $\varphi$-optimal at $R$ but not $\varphi$-optimal at $R^{\prime}$ and if $R^{\prime}$ is a profile of separable orderings, then the outcome $x$ must have fallen strictly in someone's marginal ordering. Simply put, if an equilibrium strategy profile at $R$ is to be broken at $R^{\prime}$, then the preference reversal must happen in one of the sectors. To introduce this variant of Maskin monotonicity, for any ordering $R_{i}$, outcome $x$ and sector $s$, let the weak lower contour set of $R_{i}$ and sector $s$ at $x$ be defined by $L^{s}\left(x, R_{i}\right)=\left\{\left(y^{s}, x^{s C}\right) \in X \mid x R_{i}\left(y^{s}, x^{s_{C}}\right)\right\}$ where $x^{s C}=\left(x^{1}, \cdots, x^{s-1}, x^{s+1}, \cdots, x^{\ell}\right)$. Then:

Definition 5. The SCR $\varphi: \mathcal{R}_{I} \rightarrow X$ is decomposable Maskin monotonic provided that for all $x \in X$, all $R \in \mathcal{R}_{I}$ and all $R^{\prime} \in \mathcal{R}_{I} \bigcap \mathcal{R}_{I}^{s e p}(X)$, if $x \in \varphi(R)$ and $L^{s}\left(x, R_{i}\right) \subseteq L^{s}\left(x, R_{i}^{\prime}\right)$ for all $i \in I$, for all $s \in S$, then $x \in \varphi\left(R^{\prime}\right)$.

Theorem 4. The SCR $\varphi: \mathcal{R}_{I} \rightarrow X$ is decomposable Maskin monotonic if $\varphi$ is Nash implementable in PE.

Proof. Suppose the SCR $\varphi: \mathcal{R}_{I} \rightarrow X$ is Nash implementable in PE. Then, there exists a product set of PE mechanisms $\Gamma$ such that for all $R \in \mathcal{R}_{I}$, it holds $\varphi(R)=h(N E(\Gamma, R))$. For some profile $R \in \mathcal{R}_{I}$ consider $x \in \varphi(R)$. Then, there exists a Nash equilibrium strategy profile $m \in N E(\Gamma, R)$ such that $h(m)=\left(h^{s}\left(m^{s}\right)\right)_{s \in S}=x$. Moreover, for all $i \in I$, it holds that the set of obtainable outcomes, that is, $h\left(M_{i}, m_{-i}\right)=\left\{h\left(m_{i}^{\prime}, m_{-i}\right) \in X \mid m_{i}^{\prime} \in M_{i}\right\}$, is contained in $L\left(x, R_{i}\right)$. Consider any sector $s$ and any agent $i$. Let

$$
\left(h^{s}\left(M_{i}^{s}, m_{-i}^{s}\right),\left(h^{\bar{s}}\left(m^{\bar{s}}\right)\right)_{\bar{s} \in s_{C}}\right)=\left\{\left(h^{s}\left(\bar{m}_{i}^{s}, m_{-i}^{s}\right),\left(h^{\bar{s}}\left(m^{\bar{s}}\right)\right)_{\bar{s} \in s_{C}}\right) \mid \bar{m}_{i}^{s} \in M_{i}^{s}\right\}
$$

be the set of outcomes that agent $i$ can generate by varying his own strategy for sector $s$, keeping his own strategy choices and those of other agents for sector $\bar{s} \neq s$ fixed at $m^{\bar{s}}$ and keeping the strategy choices of other agents for sector $s$ fixed at $m_{-i}^{s}$. It follows from $h\left(M_{i}, m_{-i}\right) \subseteq$ $L\left(h(m), R_{i}\right)$ that $\left(h^{s}\left(M_{i}^{s}, m_{-i}^{s}\right),\left(h^{\bar{s}}\left(m^{\bar{s}}\right)\right)_{\bar{s} \in s_{C}}\right) \subseteq L^{s}\left(h(m), R_{i}\right)$. Since agent $i$ and sector $s$ were arbitrary, it follows that

$$
\text { for all } i \in I \text { and all } s \in S:\left(h^{s}\left(M_{i}^{s}, m_{-i}^{s}\right),\left(h^{\bar{s}}\left(m^{\bar{s}}\right)\right)_{\bar{s} \in s_{C}}\right) \subseteq L^{s}\left(h(m), R_{i}\right) .
$$

Consider a profile $\bar{R} \in \mathcal{R}_{I} \bigcap \mathcal{R}_{I}^{\text {sep }}(X)$ such that

$$
\text { for all } i \in I \text { and all } s \in S: L^{s}\left(h(m), R_{i}\right) \subseteq L^{s}\left(h(m), \bar{R}_{i}\right) .
$$

Then, from (1), it follows that

$$
\text { for all } i \in I \text { and all } s \in S:\left(h^{s}\left(M_{i}^{s}, m_{-i}^{s}\right),\left(h^{\bar{s}}\left(m^{\bar{s}}\right)\right)_{\bar{s} \in s_{C}}\right) \subseteq L^{s}\left(h(m), \bar{R}_{i}\right) \text {. }
$$

Given that $\bar{R} \in \mathcal{R}_{I} \cap \mathcal{R}_{I}^{s e p}(X)$, let $\bar{R}_{i}^{s}$ be the sector-s marginal ordering induced by $\bar{R}_{i}$. Thus, from (2), we have that 
for all $i \in I$ and all $s \in S: h^{s}\left(M_{i}^{s}, m_{-i}^{s}\right) \subseteq L\left(h^{s}\left(m^{s}\right), \bar{R}_{i}^{s}\right)$, where $L\left(h^{s}\left(m^{s}\right), \bar{R}_{i}^{s}\right)$ is the weak lower contour set of $\bar{R}_{i}^{s}$ at $h^{s}\left(m^{s}\right)$. Then, for all $s \in S, m^{s} \in$ $N E\left(\Gamma^{s}, \bar{R}^{s}\right)$, and so, part (ii) of Definition 1 implies that $x^{s} \in \varphi^{s}\left(\bar{R}^{s}\right)$ for all $s \in S$. Since $\varphi$ is decomposable and $\bar{R} \in \mathcal{R}_{I} \bigcap \mathcal{R}_{I}^{\text {sep }}(X)$, it follows that $\varphi(\bar{R})=\prod_{s \in S} \varphi^{s}\left(\bar{R}^{s}\right)$, and so $x \in \varphi(\bar{R})$. Thus, $\varphi$ is decomposable Maskin monotonic.

\subsection{A characterization theorem}

In implementation theory, it is Maskin's Theorem (Maskin, 1999) that shows that when the CA faces at least three agents, a SCR is implementable in (pure-strategies) Nash equilibrium if it is Maskin monotonic and it satisfies the auxiliary condition of no veto-power. ${ }^{8}$

In the abstract Arrovian domain, the condition of no veto-power says that if an outcome is at the top of the preferences of all agents but possibly one, then it should be chosen irrespective of the preferences of the remaining agent: that agent cannot veto it. The condition of no vetopower implies two conditions. First, it implies the condition of unanimity, which states that if an outcome is at the top of the preferences of all agents, then that outcome should be selected by the SCR. Thus, as a part of sufficiency, we require a variant of unanimity, which states that if all agents agree on which outcome is best for sector $s$, then this outcome should be chosen by the $s$ th dimension of a decomposable SCR.

Definition 6. A decomposable $\operatorname{SCR} \varphi: \mathcal{R}_{I} \rightarrow X$ satisfies sector-wise unanimity provided that for all $s \in S$, all $x^{s} \in X^{s}$ and all $R^{s} \in \mathcal{D}_{I}^{s}$ if $X^{s} \subseteq L\left(x^{s}, R_{i}^{s}\right)$ for all $i \in I$, then $x^{s} \in \varphi^{s}\left(R^{s}\right)$.

Second, the condition of no veto-power implies the condition of weak no veto-power, which states that if an outcome $x$ is $\varphi$-optimal at one profile $\bar{R}$ and if the profile change from $\bar{R}$ to $R$ in a way that under the new profile an outcome $y$ that was no better than $x$ at $\bar{R}_{i}$ for some agent $i$ is weakly preferred to all outcomes in the weak lower contour set of $\bar{R}_{i}$ at $x$ according to the ordering $R_{i}$ and this $y$ is maximal for all other agents in the set $X$, then $y$ should be a $\varphi$-optimal outcome at $R$. As a part of sufficiency, we require the following adaptation of the weak no veto-power condition to our Nash implementation problems.

Definition 7. A decomposable SCR $\varphi: \mathcal{R}_{I} \rightarrow X$ satisfies sector-wise weak no veto-power provided that for all $s \in S$, all $x^{s} \in X^{s}$ and all $R^{s}, \bar{R}^{s} \in \mathcal{D}_{I}^{s}$ if $x^{s} \in \varphi^{s}\left(R^{s}\right), y^{s} \in L\left(x^{s}, R_{i}^{s}\right) \subseteq$ $L\left(y^{s}, \bar{R}_{i}^{s}\right)$ for some $i \in I$ and $X^{s} \subseteq L\left(y^{s}, \bar{R}_{j}^{s}\right)$ for all $j \in I \backslash\{i\}$, then $y^{s} \in \varphi^{s}\left(\bar{R}^{s}\right)$.

The main result of the section is also established with the aid of a domain restriction, which we now state below. Recall that $L^{s}\left(x, R_{i}\right)=\left\{\left(y^{s}, x^{s_{C}}\right) \in X \mid x R_{i}\left(y^{s}, x^{s_{C}}\right)\right\}$ where $x^{s_{C}}=$ $\left(x^{1}, \cdots, x^{s-1}, x^{s+1}, \cdots, x^{\ell}\right)$. Then:

Property 1 (P1, for short): The domain $\mathcal{R}_{I}$ satisfies $P 1$ if for all $R \in \mathcal{R}_{I}$ and all $x \in X$ there exists a profile $\bar{R} \in \mathcal{R}_{I} \bigcap \mathcal{R}_{I}^{\text {sep }}(X)$ such that for every $i \in I$ it holds that

\footnotetext{
8 Moore and Repullo (1990), Dutta and Sen (1991), Sjöström (1991) and Lombardi and Yoshihara (2013) refined Maskin's Theorem by providing necessary and sufficient conditions for a SCR to be implementable in (pure strategies) Nash equilibrium. For an introduction to the theory of implementation see Jackson (2001) and Maskin and Sjöström (2002).
} 


$$
\text { for all } s \in S: L^{s}\left(x, R_{i}\right) \subseteq L^{s}\left(x, \bar{R}_{i}\right)
$$

and that

$$
L\left(x, \bar{R}_{i}\right) \subseteq L\left(x, R_{i}\right)
$$

Basically, P1 says that for every outcome $x \in X$ and every agent $i$, a non-separable preference ranking $R_{i}$ can be part of the domain of orderings on $X$ for agent $i$ if it is coupled with a separable preference ranking $\bar{R}_{i}$ such that (a) for every sector $s$, there is a monotonic change of marginal preferences around $x^{s}$ from $R_{i}\left(x^{s_{C}}\right)$ to $\bar{R}_{i}\left(x^{s_{C}}\right)$, and (b) the change of preferences from $\bar{R}_{i}$ to $R_{i}$ is a monotonic change around $x$. Therefore, for a given profile of non-separable orderings $R$, P1 requires that one should be able to find an observationally equivalent profile of separable orderings $\bar{R}$ such that the standing of $x$ improves when preferences change from $\bar{R}$ to $R$. When P1 is violated, as shown in Example 1, there can be an agent who can secure a preferred outcome by obtaining in each sector an outcome $y^{s}$ that is worse than $x^{s}$ according the untrue environment announced by other agents. Note that P1 does not have any bite if each agent $i$ 's domain of orderings consists only of separable preference rankings. Also, note that a separable ordering $\bar{R}_{i}$ that satisfies (3) and (4) also satisfies the following property: $L^{s}\left(x, R_{i}\right)=L^{s}\left(x, \bar{R}_{i}\right)$ for each sector $s \in S$. Examples of domains satisfying P1 are provided in the next section.

The main result of this subsection can be stated as follows:

Theorem 5. Let $n \geq 3$. Suppose that the domain $\mathcal{R}_{I}$ satisfies P1. The SCR $\varphi: \mathcal{R}_{I} \rightarrow X$ is Nash implementable in PE if $\varphi$ satisfies decomposability, Maskin monotonicity, sector-wise Maskin monotonicity, decomposable Maskin monotonicity, sector-wise unanimity and sector-wise weak no veto-power.

Proof. See Appendix.

Note that the above result basically coincides with Maskin (1999)'s result when there is only one sector. Before discussing the implications of Theorem 5 in the next section, let us first show that the domain condition represented by $\mathrm{P} 1$ is indeed an indispensable requirement for our characterization result.

Example 1. $P 1$ is indispensable for Theorem 5. Let $n=3$ and $\ell=2$. Let $I=\{A, B, C\}$ and let $S=\{1,2\}$. For sector $s \in S$, let $X^{s}=\left\{x^{s}, y^{s}\right\}$ with $x^{s} \neq y^{s}$.

Suppose that agent $A$ 's domain $\mathcal{R}_{A}$ consists of the following strict orderings:

$$
\begin{aligned}
& \left(y^{1}, y^{2}\right) P_{A}\left(x^{1}, x^{2}\right) P_{A}\left(y^{1}, x^{2}\right) P_{A}\left(x^{1}, y^{2}\right) \\
& \left(x^{1}, x^{2}\right) \bar{P}_{A}\left(x^{1}, y^{2}\right) \bar{P}_{A}\left(y^{1}, x^{2}\right) \bar{P}_{A}\left(y^{1}, y^{2}\right) \\
& \left(y^{1}, y^{2}\right) \tilde{P}_{A}\left(y^{1}, x^{2}\right) \tilde{P}_{A}\left(x^{1}, y^{2}\right) \tilde{P}_{A}\left(x^{1}, x^{2}\right) .
\end{aligned}
$$

Among the listed orderings, one can check that the only ordering that is not a separable one is $P_{A}$. The marginal orderings of $P_{A}$ are as follows:

$$
\begin{aligned}
& \text { for sector } 1: \quad x^{1} P_{A}^{1}\left(x^{2}\right) y^{1} \text { and } y^{1} P_{A}^{1}\left(y^{2}\right) x^{1} \\
& \text { for sector } 2: \quad x^{2} P_{A}^{2}\left(x^{1}\right) y^{2} \text { and } y^{2} P_{A}^{2}\left(y^{1}\right) x^{2} \text {. }
\end{aligned}
$$

On the other hand, the marginal orderings of the separable orderings are as follows: 
for sector $1 \quad: \quad x^{1} \bar{P}_{A}^{1} y^{1}$ and $y^{1} \tilde{P}_{A}^{1} x^{1}$

for sector $2: x^{2} \bar{P}_{A}^{2} y^{2}$ and $y^{2} \tilde{P}_{A}^{2} x^{2}$.

By definition of the sector-s domain, we have that $\bar{P}_{A}^{s}$ and $\tilde{P}_{A}^{s}$ are elements of $\mathcal{D}_{A}^{s}$.

For the sake of simplicity, suppose that $\mathcal{R}_{i}$ for agent $i \neq A$ consists only of one strict ordering $\mathcal{R}_{i}=\left\{P_{i}\right\}$, which is as follows:

$$
\begin{array}{lll}
\text { for agent } B & : & \left(x^{1}, x^{2}\right) P_{B}\left(y^{1}, x^{2}\right) P_{B}\left(x^{1}, y^{2}\right) P_{B}\left(y^{1}, y^{2}\right) \\
\text { for agent } C & : & \left(y^{1}, y^{2}\right) P_{C}\left(y^{1}, x^{2}\right) P_{C}\left(x^{1}, y^{2}\right) P_{C}\left(x^{1}, x^{2}\right) .
\end{array}
$$

One can check that $P_{B}$ and $P_{C}$ are separable orderings on $X$, and that the marginal orderings of agents $B$ and $C$ are strict and are as follows:

for sector $1: x^{1} P_{B}^{1} y^{1}$ and $y^{1} P_{C}^{1} x^{1}$

for sector $2: x^{2} P_{B}^{2} y^{2}$ and $y^{2} P_{C}^{2} x^{2}$.

Suppose that the $\operatorname{SCR} \varphi: \mathcal{R}_{I} \rightarrow X$ satisfies all conditions of Theorem 5 but P1. Nonetheless, suppose that $\varphi$ is Nash implementable in PE. Thus, there exists a product set of PE mechanisms $\Gamma$ such that it Nash implements $\varphi$ in PE.

The profile $\left(P_{A}, P_{B}, P_{C}\right) \equiv R$ is an element of $\mathcal{R}_{I}$. Suppose that $\left(x^{1}, x^{2}\right) \in \varphi(R)$. Note that this combination would not be possible if $\mathcal{R}_{I}$ satisfied P1.

Since the $\operatorname{SCR} \varphi$ is decomposable, there exists one-dimensional SCR $\varphi^{s}$ on $\mathcal{D}_{I}^{s}$ for each $s \in S$. Given that $\varphi$ is sector-wise Maskin monotonic and, moreover, it satisfies sector-wise unanimity as well as sector-wise weak no veto-power, let

$$
\begin{aligned}
& \varphi^{1}\left(\bar{P}_{A}^{1}, P_{B}^{1}, P_{C}^{1}\right)=\left\{x^{1}\right\} \text { and } \varphi^{2}\left(\bar{P}_{A}^{2}, P_{B}^{2}, P_{C}^{2}\right)=\left\{x^{2}\right\} \\
& \varphi^{1}\left(\tilde{P}_{A}^{1}, P_{B}^{1}, P_{C}^{1}\right)=\left\{y^{1}\right\} \text { and } \varphi^{2}\left(\tilde{P}_{A}^{2}, P_{B}^{2}, P_{C}^{2}\right)=\left\{y^{2}\right\} .
\end{aligned}
$$

Furthermore, since $\varphi$ satisfies Maskin monotonicity and decomposable Maskin monotonicity, let $\varphi(R)=\left\{\left(x^{1}, x^{2}\right),\left(y^{1}, y^{2}\right)\right\}, \varphi\left(\bar{P}_{A}, P_{B}, P_{C}\right)=\left\{\left(x^{1}, x^{2}\right)\right\}$ and $\varphi\left(\tilde{P}_{A}, P_{B}, P_{C}\right)=\left\{\left(y^{1}, y^{2}\right)\right\}$. Note that part (iii) of Definition 1 is satisfied as well.

Since $\left(x^{1}, x^{2}\right) \in \varphi(R)$ and, moreover, since $\Gamma$ Nash implements $\varphi$ in PE, there exists $m \in M$ such that $h(m)=\{x\}$; that is, $h^{s}\left(m^{s}\right)=\left\{x^{s}\right\}$ for each $s \in S$. Since agent $C$ needs not find any profitable unilateral deviation and $P_{C}$ on $X$ is a separable strict ordering, it holds that $h^{s}\left(m_{-C}^{s}, M_{C}^{s}\right)=\left\{x^{s}\right\}$. Moreover, since agent $A$ also needs not find any profitable unilateral deviation from $m$, it must be the case that $h^{s}\left(m_{-A}^{s}, M_{A}^{s}\right)=\left\{x^{s}\right\}$ for at least one sector $s \in S$. Fix any of such a sector $s$. It follows that $m^{s} \in N E\left(\Gamma^{s},\left(\tilde{P}_{A}^{s}, P_{B}^{s}, P_{C}^{s}\right)\right)$ given that $h^{s}\left(m^{s}\right)=\left\{x^{s}\right\}$ is the top ranked outcome for agent $B$ according to $P_{B}^{s}$. Since $\Gamma$ Nash implements $\varphi$ in PE, part (ii) of Definition 1 implies that $x^{s} \in \varphi^{s}\left(\tilde{P}_{A}^{s}, P_{B}^{s}, P_{C}^{s}\right)$, which contradicts the fact that $\varphi^{s}\left(\tilde{P}_{A}^{s}, P_{B}^{s}, P_{C}^{s}\right)=\left\{y^{s}\right\}$. Thus, $\mathrm{P} 1$ is indispensable for Theorem 5 .

It is worth emphasizing here that P1 is not a necessary condition for implementation in PE, and so it does not provide a satisfactory answer to the question of what we loose when we adopt the practice dictated by PE mechanisms. A full answer to this question requires to provide a complete characterization of the domain of preferences on which non-trivial SCRs are Nash implementable in PE. This is an open research topic. 
A partial answer to the question is provided by Hayashi and Lombardi (2016), who provided a necessary domain condition which captures the insight provided by Example 1: No participant should be able to find a profitable deviation by obtaining a worse outcome in each sector. They have also showed the weak Pareto solution and the weak core solution cannot be Nash implemented in PE, though these solutions can be Nash implemented when the CA can design a single mechanism for the whole economy.

\section{Examples of Nash implementable SCRs in PE}

In this section, we present some implications of Theorem 5. More precisely, we consider some interesting domains that are able to accommodate some forms of complementarity. Then, we show that the sector-wise (weak) core solution and the sector-wise Vickrey-Clarke-Groves solution are Nash implementable in PE.

The example below gives a straightforward domain that satisfies P1 in matching environments with no monetary transfers. For the sake of simplicity, suppose that there are only two sectors. Items of sector 1 can be viewed as school-seats and items of sector 2 as houses. With this in mind, suppose that houses $x^{2}$ and $y^{2}$ are equally sufficiently close to respective schools $x^{1}$ and $y^{1}$. Therefore, an interpretation of type of complementarity that the example below accommodates is that agent $i$ strictly prefers the bundles that minimize the distance school-home to other available assignments and she finds the assignments that minimize the distance schoolhome, that is, $\left(x^{1}, x^{2}\right)$ and $\left(y^{1}, y^{2}\right)$, as equally good. Assignments that do not minimize the distance are viewed as equally bad.

Example 2. Non-separability of preferences in environments with no monetary transfers. In this example we provide a preference domain that satisfies P1. To this end, suppose that $S=\{1,2\}$ and that $X^{s}=\left\{x^{s}, y^{s}\right\}$, with $x^{s} \neq y^{s}$, for all $s \in S$. For any agent $i \in I$, define $\mathcal{R}_{i}$ as follows: $R_{i} \in \mathcal{R}_{i}$ if either $R_{i} \in \mathcal{R}^{s e p}(X)$ or for all $x^{1}, y^{1} \in X^{1}$ and $x^{2}, y^{2} \in X^{2}$, it holds that

$$
\left(x^{1}, x^{2}\right) I\left(R_{i}\right)\left(y^{1}, y^{2}\right) P\left(R_{i}\right)\left(y^{1}, x^{2}\right) R_{i}\left(x^{1}, y^{2}\right) .
$$

One can check that if $R_{i}$ satisfies (5), then it is not a separable ordering given that the sector-1 marginal ordering $R_{i}^{1}\left(x^{2}\right)$ differs from $R_{i}^{1}\left(y^{2}\right)$. In order to check that $\prod_{i \in I} \mathcal{R}_{i}$ satisfies $\mathrm{P} 1$, let the following separable orderings be elements of $\mathcal{R}_{i}$ :

$$
\begin{array}{lll}
\text { given }\left(x^{1}, x^{2}\right): & \left(x^{1}, x^{2}\right) P\left(\bar{R}_{i}\right)\left(y^{1}, x^{2}\right) P\left(\bar{R}_{i}\right)\left(x^{1}, y^{2}\right) P\left(\bar{R}_{i}\right)\left(y^{1}, y^{2}\right) \\
\text { given }\left(y^{1}, y^{2}\right): & \left(y^{1}, y^{2}\right) P\left(\tilde{R}_{i}\right)\left(x^{1}, y^{2}\right) P\left(\tilde{R}_{i}\right)\left(y^{1}, x^{2}\right) P\left(\tilde{R}_{i}\right)\left(x^{1}, x^{2}\right) \\
\text { given }\left(x^{1}, y^{2}\right): & \left(y^{1}, x^{2}\right) P\left(\hat{R}_{i}\right)\left(y^{1}, y^{2}\right) I\left(\hat{R}_{i}\right)\left(x^{1}, x^{2}\right) P\left(\hat{R}_{i}\right)\left(x^{1}, y^{2}\right) \\
\text { given }\left(y^{1}, x^{2}\right): & \left(x^{1}, y^{2}\right) P\left(R_{i}^{\prime}\right)\left(x^{1}, x^{2}\right) I\left(R_{i}^{\prime}\right)\left(y^{1}, y^{2}\right) P\left(R_{i}^{\prime}\right)\left(y^{1}, x^{2}\right) .
\end{array}
$$

One can now easily check that $\prod_{i \in I} \mathcal{R}_{i}$ satisfies P1.

The next result also shows that in auction/public decisions environments with monetary transfers, P1 accommodates non-separability of preferences due to income effects: If there is some change in the transfer payment of one sector, this affects how much one agent is willing to 
paylaccept for an item of another sector, and vice versa. ${ }^{9}$ To the best of our knowledge, no attention has been paid so far to this source of non-separability of preferences. This is because much of the literature on auctions and, more generally, on social decision problems with income transfers, assumes that participants' utilities are additively separable and linear in income, that is, participants have quasi-linear utilities, meaning that each participant's utility is the value of a decision or item assignment plus-or-minus the value of any income transfer that she receives or makes.

For the sake of simplicity, let us suppose that there are only two important social issues on the table, denoted by $s=1,2$, such as two large public projects. Let $D^{s}$ denote the set of potential pure social decisions for issue $s$. Let

$$
T=\left\{t \in[-\bar{t}, \infty)^{n}: \sum_{i \in I} t_{i} \leq 0\right\}
$$

denote the set of closed transfers, where the real number $\bar{t}>0$ denotes some predetermined upper-bound for payments. Let $e_{i}$ denote the initial endowment of commodity money of agent $i \in I$, which is assumed to be $e_{i} \geq 2 \bar{t}$. A social decision for issue $s$ is thus a pair $\left(d^{s}, t^{s}\right)$, where the pure decision $d^{s}$ is an element of $D^{s}$ and the vector of closed-transfer $t^{s}$ is an element of $T$. To economize on notation, let $X^{s} \equiv D^{s} \times T$.

Suppose that agent $i$ 's preferences $R_{i}$ for outcomes in $X^{1} \times X^{2}$ can be represented by a utility function $u_{i}\left(\cdot ; R_{i}\right): X^{1} \times X^{2} \rightarrow \mathbb{R}_{+}$of the form

$$
u_{i}\left(x^{1}, x^{2} ; R_{i}\right)=U_{i}\left(d^{1}, d^{2}, t_{i}^{1}+t_{i}^{2}+e_{i} ; R_{i}\right),
$$

where $U_{i}\left(\cdot ; R_{i}\right): D^{1} \times D^{2} \times \mathbb{R}_{+} \rightarrow \mathbb{R}_{+}$is strictly increasing in money. This type of utility form encompasses a wide variety of agent's preferences: separable ones and non-separable ones.

Proposition 1. Let $S=\{1,2\}$. For each $s \in S$, let $X^{s}=D^{s} \times T$, where $T$ is the set of closed transfers defined in (6). Assume that agent $i$ 's preferences belonging to $\mathcal{R}_{i}$ are represented in the form given in (7). Suppose that her willingness to paylaccept is well defined ${ }^{10}$ For each agent $i \in I$, suppose that $R_{i} \in \mathcal{R}_{i}$ satisfies the following property: For all $d^{1}, \bar{d}^{1} \in D^{1}, d^{2}, \bar{d}^{2} \in D^{2}$ and $t^{1}, t^{2} \in T$, if

\footnotetext{
9 Recall that there are two sources of non-separability of preferences. One source is represented by the complementarity of items across sectors. In general, willingness to pay for a set of items may not be equal to the sum of willingness to pay for its components. The other source is represented by income effects. The first type of non-separability is typically studied in the literature of multiple-object auctions. It is now known that non-separability across related items creates efficiency issues and strategic interaction issues. For instance, as shown by Avery and Hendershott (2000), when items are complements, running first-price auction for each item separately yields higher expected revenue than auctioning a single bundle. The reason is that a bidder who has a stronger form of preference for complementarity bids more aggressively than other bidders in each auction in order to win all the relevant items, since winning just some of them is valueless for her. Although this is optimal from the seller's viewpoint, it causes a problem of inefficiency, because it increases the probability that a bidder seeking complementarity wins only a part of the items which is valueless by itself alone, hence will resale it.

10 To assure that agent $i$ 's willingness to pay/accept is well defined, we also assume that $U_{i}$ satisfies the following property: For all $d^{1}, \bar{d}^{1} \in D^{1}$, all $d^{2}, \bar{d}^{2} \in D^{2}$, all $t^{1}, t^{2} \in T$, there exist $\bar{t}^{1}, \bar{t}^{2} \in T$ such that
}

$$
U_{i}\left(d^{1}, d^{2}, t_{i}^{1}+t_{i}^{2}+e_{i} ; R_{i}\right)=U_{i}\left(\bar{d}^{1}, \bar{d}^{2}, \bar{t}_{i}^{1}+\bar{t}_{i}^{2}+e_{i} ; R_{i}\right) .
$$




$$
\begin{aligned}
U_{i}\left(d^{1}, d^{2}, t_{i}^{1}+t_{i}^{2}+e_{i} ; R_{i}\right) & =U_{i}\left(\bar{d}^{1}, d^{2}, t_{i}^{1}+\Delta t_{i}^{1}+t_{i}^{2}+e_{i} ; R_{i}\right) \\
& =U_{i}\left(d^{1}, \bar{d}^{2}, t_{i}^{1}+t_{i}^{2}+\Delta t_{i}^{2}+e_{i} ; R_{i}\right),
\end{aligned}
$$

then

$$
U_{i}\left(\bar{d}^{1}, \bar{d}^{2}, t_{i}^{1}+\Delta t_{i}^{1}+t_{i}^{2}+\Delta t_{i}^{2}+e_{i} ; R_{i}\right)=U_{i}\left(d^{1}, d^{2}, t_{i}^{1}+t_{i}^{2}+e_{i} ; R_{i}\right) .
$$

Then, the domain $\prod_{i \in I} \mathcal{R}_{i}$ satisfies $P 1$.

Proof. Let the premises hold. Take any $\left(x^{1}, x^{2}\right) \in X^{1} \times X^{2}$ and any $R \in \prod_{i \in I} \mathcal{R}_{i}$. Since $R \in \prod_{i \in I} \mathcal{R}_{i}$ and, moreover, since each $R_{i}$ of agent $i$ has a utility representation of the form described in (7), $L\left(\left(x^{1}, x^{2}\right), R_{i}\right)$ is equivalent to $L\left(\left(d^{1}, d^{2}, t_{i}^{1}+t_{i}^{2}+e_{i}\right), U_{i}\right)$. Since for each agent $i$ the income effect is fixed at one given level and, moreover, since $R_{i}$ satisfies the above property, there exists a separable preference $R_{i}^{\prime} \in \mathcal{R}_{i}$ for agent $i$ such that the indifference surface of $U_{i}$ passing through the bundle $\left(d^{1}, d^{2}, t_{i}^{1}+t_{i}^{2}+e_{i}\right)$ coincides exactly with the indifference surface of $R_{i}^{\prime}$ through that bundle.

Before showing that the sector-wise (weak) core solution and the sector-wise Vickrey-ClarkeGroves solution are Nash implementable in PE, we need additional notation.

Definition 8. For each $R \in \mathcal{R}_{I}$ and $x \in X$, a list of profiles of marginal orderings $\left(\bar{R}^{s}\right)_{s \in S} \in$ $\prod_{s \in S} \mathcal{D}_{I}^{s}$ is equivalent to $R$ at $x$ if

for all $s \in S$ and all $i \in I: L\left(x^{s}, \bar{R}_{i}^{s}\right)=L\left(x^{s}, R_{i}^{s}\left(x^{s_{C}}\right)\right)$.

Thus, $\left(\bar{R}^{s}\right)_{s \in S}$ is equivalent to the profile $R$ at $x$ if for any sector $s$ and any agent $i$, the indifference surface of $R_{i}^{s}\left(x^{s} C\right)$ through the outcome $x^{s}$ coincides with the indifference surface of $\bar{R}_{i}^{s}$ through the same outcome. In what follows, we assume that agent $i$ 's domain of marginal preferences is rich in the following sense.

Definition 9. $\mathcal{D}_{i}^{s}$ is rich if for each $R_{i} \in \mathcal{R}_{i}, x \in X$ and $s \in S$, there exists $\bar{R}_{i}^{s} \in \mathcal{D}_{i}^{s}$ such that $L\left(x^{s}, \bar{R}_{i}^{s}\right)=L\left(x^{s}, R_{i}^{s}\left(x^{s C}\right)\right)$.

\subsection{Sector-wise (weak) core solution}

A sector-s coalitional game is a four-tuple $\left(I, X^{s}, R^{s}, v^{s}\right)$, where:

- $I$ is a finite set of agents, with $n \geq 3$.

- $X^{s}$ is a non-empty set of outcomes available from sector $s$.

- $R^{s}$ is a profile of orderings for agents on $X^{s}$.

- $v^{s}$ is a sector- $s$ characteristic function $v^{s}: 2^{N} \backslash\{\varnothing\} \rightarrow 2^{X^{s}}$, which assigns for each nonempty coalition $V$ a subset of outcomes.

Definition 10. For any sector-s coalitional game $\left(I, X^{s}, R^{s}, v^{s}\right)$, an outcome $x^{s} \in X^{s}$ is blocked by a coalition $V$ if there is $y \in v^{s}(V)$ such that $(y, x) \in P\left(R_{i}^{s}\right)$ for each $i \in V$. 
We consider a situation in which the SA knows what is feasible for each coalition, that is, the characteristic function $v^{s}$, but he does not know agents' preferences. This situation is modelled by a four-tuple $\left(I, X^{s}, \mathcal{D}_{I}^{s}, v^{s}\right)$, which we refer to as a sector-s coalitional game environment.

The sector-s core solution, denoted by $\varphi_{\text {Core }}^{s}$, is a correspondence on $\mathcal{D}_{I}^{s}$ such that for each profile $R^{s}$,

$$
\varphi_{\text {Core }}^{s}\left(R^{s}\right) \equiv\left\{x^{s} \in v^{s}(I) \mid x^{s} \text { is not blocked by any nonempty coalition } V \subseteq I\right\} .
$$

Definition 11. The SCR $\varphi_{S \text {-Core }}: \mathcal{R}_{I} \rightarrow X$ is the sector-wise core solution provided that for all $R \in \mathcal{R}_{I}$ and all $x \in X$ :

$$
x \in \varphi_{S \text {-Core }}(R) \Longleftrightarrow x \in \prod_{s \in S} \varphi_{\text {Core }}^{s}\left(\bar{R}^{s}\right)
$$

for an arbitrary list of profiles $\left(\bar{R}^{s}\right)_{s \in S} \in \prod_{s \in S} \mathcal{D}_{I}^{s}$ that is equivalent to $R$ at $x$.

In Example 2 we have provided an example of preference domain in environments with no income transfers that satisfies P1. In light of it, there are interesting domains consisting of non-separable preferences for which the sector-wise core solution is Nash implementable in PE. Formally:

Theorem 6. Take any preference domain $\mathcal{R}_{I}$ satisfying P1. Suppose that agent $i$ 's preference domain $\mathcal{D}_{i}^{s}$ is rich for each sector $s \in S$. Suppose that there are at least two sectors, $\ell \geq 2$. Let $\left(I, X^{s}, \mathcal{D}_{I}^{s}, v^{s}\right)$ be any coalitional game environment for sector $s \in S$. The sector-wise core solution $\varphi_{S \text {-Core }}: \mathcal{R}_{I} \rightarrow X$ is Nash implementable in PE.

Proof. Let the premises hold. By construction, $\varphi_{S \text {-Core }}$ satisfies decomposability. Moreover, it is well-known that sector- $s$ core solution is unanimous and Maskin monotonic. Thus, $\varphi_{S \text {-Core }}$ satisfies sector-wise Maskin monotonicity and sector-wise unanimity. We are left to show that $\varphi_{S \text {-Core }}$ satisfies Maskin monotonicity, decomposable Maskin monotonicity and sector-wise weak no veto-power. Let us first show that $\varphi_{S \text {-Core }}$ satisfies sector-wise weak no veto-power. We do it below for an arbitrary $s \in S$.

Take any $R^{s}, \hat{R}^{s} \in \mathcal{D}_{I}^{s}$ and suppose that $x^{s} \in \varphi_{\text {Core }}^{s}\left(R^{s}\right)$, that $y^{s} \in L\left(x^{s}, R_{i}^{s}\right) \subseteq L\left(y^{s}, \hat{R}_{i}^{s}\right)$ for some $i \in I$, and that $X^{s} \subseteq L\left(y^{s}, \hat{R}_{j}^{s}\right)$ for any other agent $j \in I \backslash\{i\}$. We show that $y^{s} \in$ $\varphi_{\text {Core }}^{s}\left(\hat{R}^{s}\right)$. Assume, to the contrary, that $y^{s} \notin \varphi_{\text {Core }}^{s}\left(\hat{R}^{s}\right)$.

Then, there exists a nonempty coalition $V \subseteq I$ and an outcome $z^{s} \in v^{s}(T)$ such that $z^{s} P\left(\hat{R}_{k}^{s}\right) y^{s}$ for all $k \in V$. Since the outcome $y^{s}$ is maximal for each agent $j \neq i$, it must be the case that $V=\{i\}$. Given that $z^{s} P\left(\hat{R}_{i}^{s}\right) y^{s}$, it follows that $z^{s} \notin L\left(y^{s}, \hat{R}_{i}^{s}\right)$, and so $z^{s} \notin L\left(x^{s}, R_{i}^{s}\right)$. Therefore, $z^{s} P\left(\hat{R}_{i}^{s}\right) x^{s}$, which contradicts the supposition that $x^{s}$ is a sector-s core allocation for the coalitional game $\left(I, X^{s}, R^{s}, v^{s}\right)$. Thus, $\varphi_{\text {Core }}^{s}$ satisfies weak no vetopower. We conclude that $\varphi_{S \text {-Core }}$ satisfies sector-wise weak no veto-power.

To show that the sector-wise core solution $\varphi_{S \text {-Core }}$ satisfies Maskin monotonicity, consider $x \in \varphi_{S \text {-Core }}(R)$. Then, there exists a list of profiles of marginal orderings $\left(R^{s}\right)_{s \in S} \in \prod_{s \in S} \mathcal{D}_{I}^{s}$ that is equivalent to $R$ at $x$, that is, $L\left(x^{s}, R_{i}^{s}\right)=L\left(x^{s}, R_{i}^{s}\left(x^{s_{C}}\right)\right)$ for each $i \in I$ and each $s \in S$, with $R_{i}^{s} \in \mathcal{D}_{i}^{s}$. 
Furthermore, consider any profile $\bar{R} \in \mathcal{R}_{I}$ such that for all $i \in I$, it holds that $L\left(x, R_{i}\right) \subseteq$ $L\left(x, \bar{R}_{i}\right)$. Then, for any $i \in I$ and any $s \in S$, it also holds that

for all $i \in I$, all $s \in S$ and all $y^{s} \in X^{s}:\left(x^{s}, x^{s_{C}}\right) R_{i}\left(y^{s}, x^{s_{C}}\right) \Longrightarrow\left(x^{s}, x^{s_{C}}\right) \bar{R}_{i}\left(y^{s}, x^{s_{C}}\right)$.

Since agent $i$ 's preference domain $\mathcal{D}_{i}^{s}$ is rich for each sector $s \in S$, it follows that there exists a list of profiles of marginal orderings $\left(\bar{R}^{s}\right)_{s \in S} \in \prod_{s \in S} \mathcal{D}_{I}^{s}$ that is equivalent to $\bar{R}$ at $x$, that is, $L\left(x^{s}, \bar{R}_{i}^{s}\right)=L\left(x^{s}, \bar{R}_{i}^{s}\left(x^{s_{C}}\right)\right)$ for each $i \in I$ and each $s \in S$, with $\bar{R}_{i}^{s} \in \mathcal{D}_{i}^{s}$. Moreover, by definition of $\varphi_{S \text {-Core }}$, it follows that $x^{s} \in \varphi_{\text {Core }}^{s}\left(R^{s}\right)$ for all $s \in S$. Then, for any given $s \in S$, we established that $x^{s} \in \varphi_{\text {Core }}^{s}\left(R^{s}\right)$ and $L\left(x^{s}, R_{i}^{s}\right) \subseteq L\left(x^{s}, \bar{R}_{i}^{s}\right)$ for all $i \in I$. Given that $\varphi_{\text {Core }}^{s}$ is a sector-wise Maskin monotonic SCR, we have that $x^{s} \in \varphi_{\text {Core }}^{s}\left(\bar{R}^{s}\right)$ for all $s \in S$, and so $x \in \varphi_{S \text {-Core }}(\bar{R})$. Thus, the sector-wise core solution is Maskin monotonic. Moreover, the above arguments also show that the sector-wise core solution satisfies decomposable Maskin monotonicity.

Theorem 5 implies that $\varphi_{S \text {-Core }}$ is Nash implementable in PE.

\subsection{Sector-wise VCG solution}

Let us consider the auction\public decision environment with income transfers described above. It is well-known that the Vickrey auction is a special case of the Vickrey-Clarke-Groves (VCG) mechanism, which we now proceed to define below. ${ }^{11}$ Before proceeding, let us note that complementarities as well as income effects are ruled out once participants' preferences are assumed to be separable and to have a utility representation of the form indicated in (7) — on this point, see Vives (1987) and Hayashi (2013). ${ }^{12}$ This means that each element $R_{i}^{s} \in \mathcal{D}_{i}^{s}$ has a quasi-linear utility representation

$$
u_{i}^{s}\left(x^{s}, R_{i}^{s}\right)=v^{s}\left(d^{s}, R_{i}^{s}\right)+\left(t_{i}^{s}+e_{i}\right),
$$

where $v^{s}\left(d^{s}, R_{i}^{s}\right)$ denotes the benefit that agent $i$ of type $R_{i}^{s}$ receives from a decision $d^{s} \in D^{s}$, and $t_{i}^{s}$ a payment to agents.

A sector- $s$ VCG game environment is a five-tuple $\left(I, X^{s}, \mathcal{D}_{I}^{s}, d^{s}, \tau^{s}\right)$, where:

- $I$ is a finite set of agents, with $n \geq 3$.

- $X^{s} \equiv D^{s} \times T$ is a non-empty set of outcomes available from sector-s, where $D^{s}$ denotes the set of pure decisions and $T$ is the set of closed transfers defined in (6).

- $\mathcal{D}_{I}^{s}$ is the domain of agents' quasi-linear preferences for outcomes in $X^{s}$.

- $d^{s}: \mathcal{D}_{I}^{s} \rightarrow D^{s}$ is a decision rule that prescribes the pure decision that is efficient contingent on preferences $\hat{R}^{s}$ reported by agents, that is,

$$
d^{s}\left(\hat{R}^{s}\right) \in \arg \max _{\bar{d}^{s} \in D^{s}}\left(\sum_{j \in I} v_{j}^{s}\left(\bar{d}^{s}, R_{j}^{s}\right)\right) .
$$

\footnotetext{
11 The Clarke-Groves mechanism is introduced in Clarke (1971) and Groves (1973).

12 Vives (1987) considers an increasing sequence of sets of commodities, and under certain assumptions shows that income effect on each single commodity vanishes as the number of commodity and income tend to infinity at the same rate. Hayashi (2013) considers a continuum of commodity characteristics and shows that when a commodity-described as a subset of the set of commodity characteristics — tends to be arbitrarily small the preference induced over pairs of consumption of the commodity under analysis and income transfer to be allocated to the other commodities converges to a quasi-linear one.
} 
- $\tau^{s}: \mathcal{D}_{I}^{s} \rightarrow T$ is a transfer rule that stipulates a payment to agents if $\tau_{i}^{s}\left(\hat{R}^{s}\right)>0$ (and from agents if $\tau_{i}^{s}\left(\hat{R}^{s}\right)<0$ ) contingent on preferences $\hat{R}^{s}$ reported by agents, as follows:

$$
\tau_{i}^{s}\left(\hat{R}^{s}\right)=h_{i}\left(\hat{R}_{-i}^{s}\right)-\sum_{j \in I \backslash\{i\}} v_{j}^{s}\left(d^{s}\left(\hat{R}^{s}\right), R_{j}^{s}\right),
$$

where $h_{i}(\cdot)$ is an arbitrary function that is independent of agent $i$ 's report.

The outcome $\left(d^{s}, t^{s}\right) \in X^{s}$ is a sector-s VCG outcome of the VCG game $\left(I, X^{s}, R^{s}, d^{s}, \tau^{s}\right)$ if $d^{s}=d\left(R^{s}\right)$ and $t^{s}=\tau^{s}\left(R^{s}\right)$. The sector-s VCG solution, denoted by $\varphi_{V C G}^{s}$, is a correspondence on $\mathcal{D}_{I}^{s}$ such that for each profile $R^{s}$,

$$
\varphi_{V C G}^{s}\left(R^{s}\right) \equiv\left\{x^{s} \in X^{s} \mid x^{s}=\left(d^{s}\left(R^{s}\right), \tau^{s}\left(R^{s}\right)\right)\right\} .
$$

Definition 12. The SCR $\varphi_{S-V C G}: \mathcal{R}_{I} \rightarrow X$ is the sector-wise VCG solution if for each $R \in \mathcal{R}_{I}$,

$$
x \in \varphi_{S-V C G}(R) \Longleftrightarrow x \in \prod_{s \in S} \varphi_{V C G}^{s}\left(\bar{R}^{s}\right)
$$

for an arbitrary list of profiles $\left(\bar{R}^{s}\right)_{s \in S} \in \prod_{s \in S} \mathcal{D}_{I}^{s}$ that is equivalent to $R$ at $x$.

The main result of this subsection can then be stated as follows.

Theorem 7. Take any preference domain $\mathcal{R}_{I}$ satisfying $P 1$. Suppose that agent $i$ 's preference domain $\mathcal{D}_{i}^{s}$ is rich for each sector $s \in S$. Suppose that there are at least two sectors, $\ell \geq 2$. Let $\left(I, X^{s}, \mathcal{D}_{I}^{s}, d^{s}, \tau^{s}\right)$ be any VCG game environment for sector $s \in S$. The sector-wise VCG solution $\varphi_{S-V C G}: \mathcal{R}_{I} \rightarrow X$ is Nash implementable in PE.

Proof. Let the premises hold. By construction, $\varphi_{S-V C G}$ satisfies decomposability. Moreover, it is well-known that sector-s VCG solution is Maskin monotonic and a unanimous SCR. Moreover, sector- $s$ VCG solution satisfies weak no veto-power vacuously. Thus, the sector-wise VCG solution $\varphi_{S-V C G}$ satisfies sector-wise Maskin monotonicity, sector-wise weak no veto-power and sector-wise unanimity. Reasoning like that used in the proof of Theorem 6 shows that $\varphi_{S-V C G}$ satisfies Maskin monotonicity, as well as decomposable Maskin monotonicity. Then, Theorem 5 implies that $\varphi_{S-V C G}$ is Nash implementable in PE.

\section{Concluding remarks}

A product set of PE mechanisms is a mechanism in which its participants are constrained to submit their rankings to sector authorities separately and, moreover, sector authorities cannot communicate with each other, due to misspecification by the CA that preferences are separable or due to technical/institutional constraints. Therefore, a key property of a single PE mechanism is that participants are required to behave as if they had separable preferences.

We identify a set of necessary conditions for the implementation of SCRs via a product set of PE mechanisms, that is, for the implementation in PE. Furthermore, under mild auxiliary conditions, reminiscent of Maskin's Theorem (1999), we have also shown that they are sufficient for the implementation in PE. 
We conclude by discussing future research directions. The first thing to come next will be to quantify how much we lose by the type of misspecification considered in this paper. Theoretical, empirical and experimental studies will be helpful there.

It is also worth investigating what can be implemented when an incomplete yet not negligible communication is allowed among SAs, while the central designer has to make some modelling choice about how SAs communicate.

Another direction will be to study how we can improve the mechanism in a sector while keeping fixed the mechanisms in other sectors and, given such change, how we can improve the mechanism in another sector while keeping fixed those in other sectors, and so on. There is no obvious way to do it because under general equilibrium effects it is not obvious whether or not a change regarded as an "improvement" from the point of view of PE mechanism design is indeed an improvement. That research direction will answer the question of how we should change the PE mechanism in an improving manner.

\section{Appendix}

\subsection{Proof of Theorem 5}

Let the premises hold. The proof is based on the construction of a product set of PE mechanisms $\Gamma=\left(\Gamma^{s}\right)_{s \in S}$, where sector-s PE mechanism, $\Gamma^{s}=\left(M^{s}, h^{s}\right)$, is a canonical mechanism.

\section{Sector $s \in S$ PE mechanism:}

Agent $i$ 's message space is defined by ${ }^{13}$

$$
M_{i}^{s}=\mathcal{D}_{I}^{s} \times X^{s} \times \mathcal{Z}_{+},
$$

where $\mathcal{Z}_{+}$is the set of nonnegative integers. Thus, agent $i$ 's strategy consists of an outcome in $X^{s}$, a profile of orderings and a nonnegative integer. Thus, a typical strategy played by agent $i$ is denoted by $m_{i}^{s}=\left(\left(R^{s}\right)^{i},\left(x^{s}\right)^{i}, z^{i}\right)$. The message space of agents is the product space

$$
M^{s}=\prod_{i \in I} M_{i}^{s}
$$

with $m^{s}$ as a typical strategy profile. The outcome function $h^{s}$ is defined with the following three rules:

Rule 1: If $m_{i}^{s}=\left(\left(R^{s}\right)^{i},\left(x^{s}\right)^{i}, 0\right)=\left(\bar{R}^{s}, x^{s}, 0\right)$ for each agent $i \in I$ and $x^{s} \in \varphi^{s}\left(\bar{R}^{s}\right)$, then $h^{s}\left(m^{s}\right)=x^{s}$.

Rule 2: If $n-1$ agents play $m_{j}^{s}=\left(\bar{R}^{s}, x^{s}, 0\right)$ with $x^{s}=\varphi^{s}\left(\bar{R}^{s}\right)$, but agent $i$ plays $m_{i}^{s}=$ $\left(\left(R^{s}\right)^{i},\left(x^{s}\right)^{i}, z^{i}\right) \neq\left(\bar{R}^{s}, x^{s}, 0\right)$, then we can have two cases:

1. If $x^{s} \bar{R}_{i}^{s}\left(x^{s}\right)^{i}$, then $h^{s}\left(m^{s}\right)=\left(x^{s}\right)^{i}$.

2. If $\left(x^{s}\right)^{i} P\left(\bar{R}_{i}^{s}\right) x^{s}$, then $h^{s}\left(m^{s}\right)=x^{s}$.

$\overline{13}$ Note that $\mathcal{D}_{i}^{s}$ is nonempty for each agent $i \in I$ since $\mathrm{P} 1$ holds. 
Rule 3: Otherwise, an integer game is played: identify the agent who plays the highest integer (if there is a tie at the top, pick the agent with lowest index among them.) This agent is declared the winner of the game and the alternative implemented is the one she selects.

Since $\varphi$ is decomposable, there exists a sequence $\left(\varphi^{s}\right)_{s \in S}$ of one-dimensional SCRs, where $\varphi^{s}: \mathcal{D}_{I}^{s} \rightarrow X^{s}$ for each $s \in S$. Also, note that the proof of part (ii) of Definition 1 follows very closely the proof of Repullo (1987, pp. 40-41) given that $\varphi^{s}$ satisfies sector-wise Maskin monotonicity, sector-wise weak no veto-power and sector-wise unanimity. To complete the proof, we show that part (i) and part (iii) of Definition 1 are satisfied as well. Thus, let us fix any $R \in \mathcal{R}_{I}$.

Nothing has to be proved if $R$ is a profile of separable orderings. The reason is that $N E(\Gamma, R)=\prod_{s \in S} N E\left(\Gamma^{s}, R^{s}\right)$ and that part (ii) and part (iii) of Definition 1 hold. Therefore, let us suppose that $R$ is not a profile of separable orderings.

Let us first show that $h(N E(\Gamma, R)) \subseteq \varphi(R)$. Take any $x \in h(N E(\Gamma, R))$. Then, there exists $m \in N E(\Gamma, R)$ such that $h(m)=\left(h^{s}\left(m^{s}\right)\right)_{s \in S}=x$. Then, given that $m \in N E(\Gamma, R)$, for each agent $i \in I$ it holds that

$$
\text { for each } s \in S: h^{s}\left(m^{s}\right) R_{i}^{s}\left(\left(h^{\hat{s}}\left(m^{\hat{s}}\right)\right)_{\hat{s} \in S \backslash\{s\}}\right) h^{s}\left(\hat{m}_{i}^{s}, m_{-i}^{s}\right) \text { for each } \hat{m}_{i}^{s} \in M_{i}^{s} \text {. }
$$

Given that $\mathcal{R}_{I}$ satisfies $\mathrm{P} 1$, there exists a profile $\bar{R} \in \mathcal{R}_{I} \bigcap \mathcal{R}_{I}^{\text {sep }}(X)$ such that for all $i \in I$, it holds that

$$
\text { for all } s \in S: L^{s}\left(h(m), R_{i}\right) \subseteq L^{s}\left(h(m), \bar{R}_{i}\right)
$$

and that

$$
L\left(h(m), \bar{R}_{i}\right) \subseteq L\left(h(m), R_{i}\right) .
$$

Then, from (11) and (12) and from the fact that $\bar{R} \in \mathcal{R}_{I} \bigcap \mathcal{R}_{I}^{\text {sep }}(X)$, it follows that for all $i \in I$ and all $s \in S$ it holds that

$$
h^{s}\left(m^{s}\right) \bar{R}_{i}^{s} h^{s}\left(\tilde{m}_{i}^{s}, m_{-i}^{s}\right) \text { for all } \tilde{m}_{i}^{s} \in M_{i}^{s},
$$

and so

$$
\text { for all } s \in S: m^{s} \in N E\left(\Gamma^{s}, \bar{R}^{s}\right) \text {. }
$$

Part (ii) of Definition 1 and decomposability imply that $h(m) \in \varphi(\bar{R})$. Since the SCR $\varphi$ is Maskin monotonic and since, moreover, (13) holds for every agent, it follows that $h(m) \in \varphi(R)$. This completes the proof that $h(N E(\Gamma, R)) \subseteq \varphi(R)$. Note that the preceding arguments also show that part (iii) of Definition 1 is met as well.

For the converse, suppose that $x \in \varphi(R)$. Given that the $\mathcal{R}_{I}$ satisfies $\mathrm{P} 1$, it follows that there exists a profile $\bar{R} \in \mathcal{R}_{I} \bigcap \mathcal{R}_{I}^{\text {sep }}(X)$ such that for all $i \in I$, it holds that

$$
\text { for all } s \in S: L^{s}\left(x, R_{i}\right) \subseteq L^{s}\left(x, \bar{R}_{i}\right)
$$

and that

$$
L\left(x, \bar{R}_{i}\right) \subseteq L\left(x, R_{i}\right) .
$$

Since $x \in \varphi(R)$ and since, moreover, (14) holds for each agent $i$ and each sector $s$, decomposable Maskin monotonicity implies that $x \in \varphi(\bar{R})$. Furthermore, given that $\bar{R} \in$ $\mathcal{R}_{I} \cap \mathcal{R}_{I}^{\text {sep }}(X)$, decomposability implies $\varphi(\bar{R})=\prod_{s \in S} \varphi^{s}\left(\bar{R}^{s}\right)$, where $\bar{R}^{s} \in \mathcal{D}_{I}^{s}$ is the profile 
of sector-s marginal orderings induced by $\bar{R}$. Part (ii) of Definition 1 implies that $\varphi(\bar{R})=$ $\prod_{s \in S} h^{s}\left(N E\left(\Gamma^{s}, \bar{R}^{s}\right)\right)$. Moreover, given that $N E(\Gamma, \bar{R})=\prod_{s \in S} h^{s}\left(N E\left(\Gamma^{s}, \bar{R}^{s}\right)\right)$, we have that $\varphi(\bar{R})=h(N E(\Gamma, \bar{R}))$. Thus, there exists $m \in M$ such that $h(m)=x$ and that

$$
\text { for all } i \in I:\left\{h\left(m_{i}^{\prime}, m_{-i}\right) \in X \mid m_{i}^{\prime} \in M_{i}\right\} \subseteq L\left(x, \bar{R}_{i}\right) .
$$

Finally, given that (15) holds, it follows that

$$
\text { for all } i \in I:\left\{h\left(m_{i}^{\prime}, m_{-i}\right) \in X \mid m_{i}^{\prime} \in M_{i}\right\} \subseteq L\left(x, R_{i}\right) .
$$

We have that $h(m)=x \in h(N E(\Gamma, R))$. Thus, we have established part (i) of Definition 1 .

\section{References}

Avery, C., Hendershott, T., 2000. Bundling and optimal auctions of multiple products. Rev. Econ. Stud. 67 (3), $483-497$. Barberà, S., Sonnenschein, H., Zhou, L., 1991. Voting by committees. Econometrica 59, 595-609.

Clarke, E.H., 1971. Multipart pricing of public goods. Public Choice 11, 17-33.

Dutta, B., Sen, A., 1991. A necessary and sufficient condition for two-person Nash implementation. Rev. Econ. Stud. 58, 121-128.

Groves, T., 1973. Incentives in teams. Econometrica 41, 617-631.

Hayashi, T., 2013. Smallness of a commodity and partial equilibrium analysis. J. Econ. Theory 48, 279-305.

Hayashi, T., Lombardi, M., 2016. Implementation in partial equilibrium. Available at SSRN https://ssrn.com/abstract= 2718444.

Jackson, M.O., 2001. A crash course in implementation theory. Soc. Choice Welf. 18, 655-708.

Le Breton, M., Sen, A., 1999. Separable preferences, strategyproofness and decomposability. Econometrica 67, 605-628.

Lombardi, M., Yoshihara, N., 2013. A full characterization of Nash implementation with strategy space reduction. Econ. Theory 54, 131-151.

Maskin, E., 1999. Nash equilibrium and welfare optimality. Rev. Econ. Stud. 66, 23-38.

Maskin, E., Sjöström, T., 2002. Implementation theory. In: Arrow, K., Sen, A.K., Suzumura, K. (Eds.), Handbook of Social Choice and Welfare. Elsevier Science, Amsterdam. 2002. pp. 237-288.

Moore, J., Repullo, R., 1990. Nash implementation: a full characterization. Econometrica 58, 1083-1100.

Repullo, R., 1987. A simple proof of Maskin's theorem on Nash implementation. Soc. Choice Welf. 4, 39-41.

Shapley, L., Scarf, H., 1974. On core and indivisibility. J. Math. Econ., 23-37.

Sjöström, T., 1991. On the necessary and sufficient conditions for Nash implementation. Soc. Choice Welf. 8, 333-340.

Vickrey, W., 1961. Counterspeculation, auctions, competitive sealed tenders. J. Finance 16, 8-37.

Vives, X., 1987. Small income effects: a Marshallian theory of consumer surplus and downward sloping demand. Rev. Econ. Stud. 54, 87-103. 